\title{
Repeatability Estimation for Vibration Measurement Results of Power Transformer Core
}

\author{
S. Borucki*, A. Cichon, J. Skubis And T. Boczar \\ Faculty of Electrical Engineering, Automatic Control and Computer Science, Opole University of Technology \\ Prószkowska 76, 45-758 Opole, Poland
}

\begin{abstract}
The subject matter of this paper refers to the diagnostics of a transformer core, based on measurements and analysis of vibroacoustic signals registered during their normal operation. The paper presents results of the research aimed to evaluate the repeatability of vibration measurements for power transformer core. Recording of vibroacoustic signals was performed under laboratory conditions on dry-insulated transformers. The study concerned evaluation of changes in vibroacoustic indicators of the transformer core during operation with a turned and loosened core. Conclusions from the measurement results were drawn on the basis of the parametric test of significance derived from the variance analysis for many means of single classification basing on the Fisher-Snedecor F-distribution.
\end{abstract}

DOI: 10.12693/APhysPolA.125.1299

PACS: 43.40.-r, 43.40.Vn, 43.58.Wc

\section{Introduction}

Vibroacoustic methods for testing the technical condition of devices are more widely used in various sectors of the economy. This is due to the fact that diagnosis based on measurement and analysis of mechanical vibrations do not require permanent or temporary damage to the structure of test unit. Vibroacoustic methods are currently used in diagnosis of rotating machines, as well as for devices operating statically [1]. With regard to transformers, this method is more widely used, particularly in relation to high voltage block and network units. Linkage results obtained during vibroacoustic measurements to other test results (gas chromatography, measurements of frequency response) provides information on the condition of the test transformer core [2-4]. Magnetostriction and electrodynamic forces influence the core cause of its vibration. Once metal plate packets of the magnetic circuit are loosened, then vibroacoustic signals registered on the core or surface of the tank have significantly higher amplitude and are characterised by energetic participation of frequency components other than in the case of transformer without a defect. Tracking RMS value changes and performing amplitude growth analysis of particular harmonic vibrations allows then monitoring and assessment of the core technical condition without the need of power interruptions in the test transformer.

This paper presents detailed results from the repeatability assessment of measurement results obtained during laboratory tests of vibroacoustic signals for a dry-type transformer. Assessment of the repeatability for the results obtained is shown on an example of statistical analysis of root mean square (RMS) value of vibration

*corresponding author; e-mail: s.borucki@po.opole.pl, s.borucki@gmail.com acceleration within the band of $5-2500 \mathrm{~Hz}$, which has been designated for transformer operating with turned and loosened core. Measurement method and methodology for modeling damages in magnetic circuit of the test transformer are presented in $[5,6]$.

\section{Research methodology}

Measurements and repeatability analysis of measured mechanical vibrations in the transformer core were conducted in the Laboratory of Insulation System Diagnostics of the Opole University of Technology. The monitored unit, its general view is presented in Fig. 1, is of following parameters: ET3S-15 type, power - $15 \mathrm{kVA}$, primary voltage $-400 \mathrm{~V}$, secondary voltage $-24 \mathrm{~V}$, group of connections - Yy, type of insulation - dry, production year -2006 .

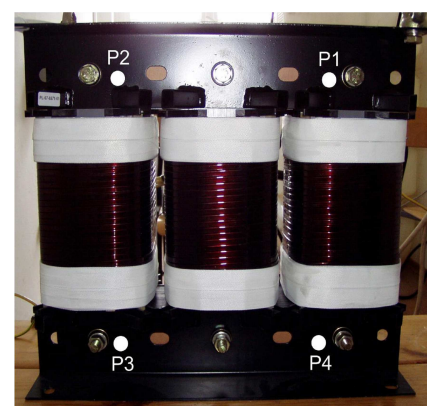

Fig. 1. General view of the test transformer with indicated measurement points.

Selecting dry-type transformer for the experiment allowed for "relatively" simple modeling of the damage occurring in its magnetic circuit. This modeling was implemented by changing the torsional torque of press cramps between upper and lower yoke beams. At the 
same time, by using four measuring transducers, simultaneous assessment of the technical condition of the magnetic circuit of the transformer was possible to be performed simultaneously in several areas. Measurements were performed by using measuring apparatus characterized in $[5,7]$.

Since the vibrations generated by the transformer were registered repeatedly, new repeatability analysis of the measured data was performed. The analysis was performed on the basis of RMS values of vibration acceleration within the band of $5-2500 \mathrm{~Hz}$, which was designated for operation of the transformer with turned and loosened core. The paper presents only selected results of statistical analysis, which was performed for vibroacoustic signals measured at $\mathrm{P} 1$ point.

In order to perform analyses of statistical repeatability assessments of measurement results for each population contain the obtained results for operating the transformer without any defect and with modeled damage. There were obtained populations comprising on average of approximately 100 samples. Statistical analysis was performed using numerical procedures of Statistica, Statgraphics Plus and MS Excel software.

Findings from the obtained measurement results were derived from parametric significance test based on analysis of variance (ANOVA) on the basis of the F-distribution $[8,9]$, which on the initial stage required checking if results in the tested populations are of normal distribution and if their variations are equal, wherein they do not have to become known. For this purpose, nonparametric $H_{0}$ null hypothesis was adopted, that is the designated RMS values of vibration acceleration have distribution which can be described using $F(x)$ distribution function, corresponding to the density function of normal distribution formulated by Gauss-Laplace. While, in the $H_{1}$ alternative hypothesis, it was assumed that the obtained measurements are not part of the set of normal distribution functions. Adopted hypothesis can be formed as

$$
H_{0}: F(x) \in \Omega, \quad H_{1}: F(x) \notin \Omega,
$$

where $\Omega$ is the class of all normal distribution functions.

Verification of the assumed null hypothesis was performed using the non-parametric for testing compliance - $\chi^{2}$ (Pearson's chi-squared test), which is most frequently used for equivalence hypothesis testing of measurement probability density at assumed theoretical probability density [10-12]. Assessment of the distance between distribution functions was conducted using statistic measures

$$
\chi^{2}=\sum_{i=1}^{r} \frac{\left(n_{i}-n p_{i}\right)^{2}}{n p_{i}},
$$

where $r$ - number of class ranges, $n=\sum_{i=1}^{r} n_{i}-$ population size, $p_{i}$ - probability that the future takes a value within the $i$-th class range, for distribution consistent with $H_{0}, n p_{i}=\hat{n}_{i}-$ (expected) number of theoretical units, which should be included in the $i$-th class range by assuming that the feature has distribution con- sistent with the hypothetical one, while theoretical numbers shall not be low and should satisfy the condition $n p_{i}=\hat{n}_{i} \geq 5 ; n_{i}$ - empirical population of the $i$-th class range.

Subsequently, the $\chi^{2}$ statistics, calculated according to the formula (2) were compared with the $\chi_{\alpha}^{2}$ critical value, which was read from chi-square distribution table, at a set level of significance $\alpha$ and computed number of degrees of freedom. Critical region for this test was built upward to satisfy the relation

$$
P\left(\chi^{2} \geq \chi_{\alpha}^{2}\right)=\alpha,
$$

where $\chi_{\alpha}^{2}$ is critical value, being read from the $\chi^{2}$ distribution for $r-s-1$ degrees of freedom and $P=\alpha$ [12].

If $\chi^{2} \geq \chi_{\alpha}^{2}$, then the $H_{0}$ hypothesis should be rejected, because the difference between the empirical and hypothetical distribution was statistically weak. Otherwise, there was reason for its rejection. Calculations were conducted for a significance level of $\alpha=0.05$, usually adopted for this type of analyses [12].

Assuming equality of variances, parametric multivariate analysis of variance (MANOVA) was performed, which assumes the following hypotheses: $H_{0}: m_{1}=$ $m_{2}=\ldots=m_{i}=m_{k}$, where $m_{i}$ is the mean value of $i$-th population, $H_{1}$ : not all mean values are equal.

To verify such formulated $H_{0}$ hypothesis, significance test based on $F$ statistics was used, expressed by the formula (4):

$$
F=\frac{\hat{S}_{1}^{2}}{\hat{S}_{2}^{2}},
$$

where

$$
\left\{\begin{array}{l}
\hat{S}_{1}^{2}=\frac{\sum_{i=1}^{k}\left(\bar{x}_{i}-\overline{\bar{x}}\right)^{2} n_{i}}{k-1} \\
\hat{S}_{2}^{2}=\frac{\sum_{i=1}^{k} \sum_{j=1}^{n_{i}}\left(x_{i j}-\bar{x}_{i}\right)^{2}}{n-k} \\
\bar{x}_{i}=\frac{1}{n_{i}} \sum_{j=1}^{n_{i}} x_{i j} \quad \text { for } i=1,2, \ldots, k \\
\overline{\bar{x}}=\frac{1}{n} \sum_{i=1}^{k} \sum_{j=1}^{n_{i}} x_{i j} \quad \text { where } n=\sum_{i=1}^{k} n_{i},
\end{array}\right.
$$

where $\overline{\bar{x}}$ - total arithmetic mean, $\bar{x}_{i}$ - group means, $\hat{S}_{1}^{2}, \hat{S}_{2}^{2}$ - variances [12].

Assuming that the $H_{0}$ hypothesis is true, the $F$ statistics has an $\mathrm{F}$ distribution (Fisher-Snedecor distribution) with $k-1$ and $n-k$ degrees of freedom. Subsequently, the derived $F$ value was compared with the $F_{\alpha}$ critical value read from the Fisher-Snedecor distribution table, for the adopted significance level of $\alpha$ and an adequate value of $k-1$ and $n-k$ degrees of freedom, while the equation shall be satisfied: $P\left\{F>F_{\alpha}\right\}=\alpha$. If $F>F_{\alpha}$ inequality is obtained as a result of comparison, then the $H_{0}$ hypothesis on equality of means in the analysed populations should be rejected. Whereas, if $F<F_{\alpha}$, then there is no reason to reject the $H_{0}$ hypothesis stating that all means are equal. If $F<1$, then there is no reason to reject the null hypothesis without comparing it with $F_{\alpha}$. Rejecting the $H_{0}$ hypothesis means proving the significance of the distribution on this population. Otherwise, it can be concluded that all groups are equivalent in terms of the examined attribute [12]. 


\section{Test result analysis}

This section provides selected analysis results of the RMS value of vibration acceleration for turned and loosened transformer core. In an analogous manner, the repeatability of results obtained for the other vibroacoustic parameters designated for various operating states of the magnetic circuit in the tested unit were checked.

Numerical values used to verify the $H_{0}$ hypothesis, which assumes normal distribution of vibroacoustic signals, determined at $\mathrm{P} 1$ point for turned and loosened core were presented in Tables I and II. Columns of the table include, respectively: number of class ranges, RMS value ranges of vibration acceleration corresponding to a given class, population size and their $\chi^{2}$ type distributions.

TABLE I

Distribution board of $\chi^{2}$ type, designated for RMS value of vibration acceleration for transformer core turned core.

\begin{tabular}{c|c|c|c}
\hline \hline Class range & $A\left[\mathrm{~cm} / \mathrm{s}^{2}\right]$ & $\begin{array}{c}\text { Number of } \\
\text { observations }\end{array}$ & $\chi^{2}$ \\
\hline 1 & $\leq 1.10$ & 0 & 0 \\
2 & 1.20 & 13 & 0.69 \\
3 & 1.30 & 26 & 0.52 \\
4 & 1.40 & 32 & 0.39 \\
5 & 1.50 & 11 & 1.05 \\
6 & 1.60 & 10 & 1.42 \\
7 & 1.70 & 5 & 1.12 \\
8 & 1.80 & 2 & 1.90 \\
9 & 1.90 & 1 & 0.98 \\
10 & unlimited & 0 & 0 \\
\hline total & & 100 & 8.07
\end{tabular}

Results from the performed verifications are shown in Table III that contain comparison between the total value of $\chi^{2}$ type distribution, calculated for the designated vibration indicators and the critical value of $\chi_{\alpha}^{2}$, read from the distribution table for the degrees of freedom correction and the given value of significance level of $\alpha=0.05$.

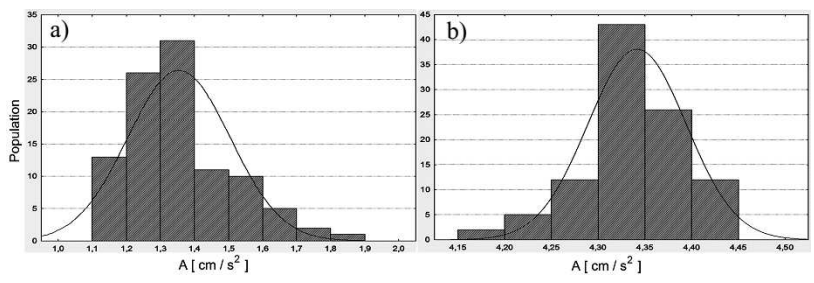

Fig. 2. Probability density function designated for the RMS value of vibration acceleration for the transformer core: (a) with a turned core, (b) with a loosened core.

For general population representing the examined RMS values of vibration acceleration there were presented waveforms of the probability density function corresponding to the Gaussian and Laplace normal distri-
TABLE II

Distribution board of $\chi^{2}$ type, designated for RMS value of vibration acceleration for transformer core loosened core.

\begin{tabular}{c|c|c|c}
\hline \hline Class range & $A\left[\mathrm{~cm} / \mathrm{s}^{2}\right]$ & $\begin{array}{c}\text { Number of } \\
\text { observations }\end{array}$ & $\chi^{2}$ \\
\hline 1 & $\leq 4.16$ & 0 & 0.05 \\
2 & 4.18 & 1 & 0 \\
3 & 4.20 & 1 & 0.04 \\
4 & 4.23 & 1 & 0.22 \\
5 & 4.25 & 3 & 0.19 \\
6 & 4.27 & 3 & 0.06 \\
7 & 4.29 & 6 & 0.34 \\
8 & 4.31 & 11 & 0.25 \\
9 & 4.33 & 18 & 0.20 \\
10 & 4.35 & 19 & 0.01 \\
11 & 4.37 & 15 & 0.01 \\
12 & 4.39 & 9 & 0.01 \\
13 & 4.42 & 6 & 0.11 \\
14 & 4.44 & 3 & 0.20 \\
15 & 4.46 & 4 & 0.31 \\
16 & unlimited & 0 & 0.32 \\
\hline total & & 100 & 2.27
\end{tabular}

TABLE III

Comparative tabulation of index values designated for the results obtained and read from the test table of $\chi^{2}$ and $F$ for RMS value of vibration acceleration for transformer core.

\begin{tabular}{c|c|c|c|c|c|c}
\hline \hline \multirow{2}{*}{$\begin{array}{c}\text { Transformer } \\
\text { core }\end{array}$} & \multicolumn{5}{|c}{$\begin{array}{c}\text { Root mean square value of vibration } \\
\text { acceleration }\left[\mathrm{cm} / \mathrm{s}^{2}\right]\end{array}$} \\
\cline { 2 - 7 } & $\begin{array}{c}\text { Mean } \\
\text { value }\end{array}$ & $\begin{array}{c}\text { Standard } \\
\text { deviation }\end{array}$ & $\chi^{2}$ & $\chi_{\alpha}^{2}$ & $F$ & $F_{\alpha}$ \\
\hline turned core & 1.35 & 0.15 & 8.07 & 15.51 & 1.55 & 2.53 \\
loosened core & 4.43 & 0.05 & 2.27 & 15.51 & 0.18 & 2.53
\end{tabular}

bution (Fig. 2), being matched with histograms showing empirical distribution of the measured signals.

Since the inequalities $\chi^{2}=8.07<15.51=\chi_{\alpha}^{2}$ and $\chi^{2}=2.27<15.51=\chi_{\alpha}^{2}$ are satisfied, there is no reason to reject the $H_{0}$ hypothesis stating that distribution of RMS values of vibration acceleration for the (turned and loosened) power transformer is normal, with $5 \%$ fault tolerance.

\section{Summing-up}

Since the inequality was satisfied: $\chi^{2}<\chi_{\alpha}^{2}$, there would be no reason to reject the $H_{0}$ hypothesis, which states that all variances of the vibration measurements for the turned and loosened transformer core while maintaining fault tolerance of 5 -percent. For each of the analysed cases, the inequality $F<F_{\alpha}$ was also satisfied, which suggests that there was no reason to reject the $H_{0}$ hypothesis claiming that all RMS values of vibration acceleration are equal. The assumed null hypothesis is true, which proves that for two extremely different 
conditions of the test transformer core, the repeatability of measurements of RMS value of vibration acceleration occurs with 5 percent fault tolerance. Tests conducted for the other vibroacoustic parameters designated in the time-frequency domain had the same test result.

\section{Acknowledgments}

The work was financed with the means from the National Research and Development Center within LIDER program.

\section{References}

[1] J. Morel, Vibrations of Machines and Diagnostics of their Mechanical State, Erreur Perimes Eyrolles Pub., France 1992 (in French).

[2] C. Bartoletti, M. Desiderio, et al. IEEE Trans. Power Deliv. 19, 221 (2004).

[3] J. Shengchang, L. Youngfen, L. Yanming, IEEE Trans. Power Deliv. 21, 1981 (2006).

[4] M. Duval, IEEE Electr. Ins. Mag. 24, 7 (2008).
[5] S. Borucki, IEEE Trans. Power Deliv. 27, 670 (2012).

[6] S. Borucki, Acta Phys. Pol. A 120, 571 (2011).

[7] T. Boczar, D. Wotzka, Acta Phys. Pol. A 122, 850 (2012).

[8] M. Dobosz, The Computer-Assisted Statistical Analysis of the Results, Publishing House of EXIT, Warsaw 2001 (in Polish).

[9] S. Ostasiewicz, Z. Rusnak, U. Siedlecka, Statistics Elements of the Theory and Tasks, Wydawnictwo AE, Wrocław 1997 (in Polish).

[10] J. Greń, Mathematical Statistics, Models and Tasks, PWN, Warszawa 1994 (in Polish).

[11] M. Sobczyk, Statistics, Wydawnictwo Naukowe PWN, Warsaw 2002 (in Polish).

[12] T. Boczar, Possible Application for Description of Acoustic Emission Signals Generated by PDs in Statistical Analysis and Digital Signal Processing Methods, Publishing House of the Opole University of Technology, SiM Issue 146, Opole 2003 (in Polish). 